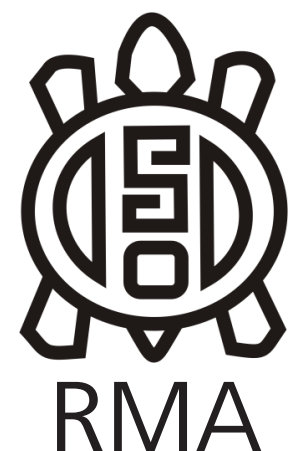

Arqueología

\title{
"Recorriendo la Graciana". Aspectos metodológicos y resultados del trabajo de campo del sitio LRA, Valle de Ambato, Catamarca, Argentina
}

\author{
"Traveling the Graciana". Methodological aspects and results of \\ the field work of the LRA site
}

Melisa Rodríguez Oviedo

IDACOR, Museo de Antropología, Universidad Nacional de Córdoba. Hipólito Yrigoyen 174 (CP 5000), Córdoba, Argentina. Correo: melisa.roviedo@gmail.com

\begin{abstract}
Resumen
En esta nota daremos cuenta de los trabajos realizadas en el sitio La Rinconada Arriba (de ahora en adelante LRA) ubicado en el Valle de Ambato, Catamarca, Argentina. Este sitio fue estudiado con el propósito de profundizar los trabajos realizados en la ladera oriental del valle (sierra de La Graciana). Con el fin de concretar este objetivo se realizaron tareas de campo (prospección y relevamiento) durante los meses de Octubre y Noviembre de 2014. Como resultado de estas actividades se lograron registrar e identificar diversos tipos de estructuras (recintos, corrales y canales), de los cuales se recuperaron materiales cerámicos y líticos en superficie. Esta labor permitió identificar un sector destinado principalmente a prácticas agrícolas, que a priori parecieran romper con la configuración productiva propuesta previamente para el valle. La información obtenida producto de este trabajo permite plantear que LRA estuvo enfocada exclusivamente en la producción agrícola y que constituyó una particularidad dentro del modelo agro-pastoril propuesto para la zona en estudio. De este modo, es posible pensar a este sistema no como un todo no homogéneo, sino con variantes que pueden responder a cuestiones sociales, políticas, ambientales y/o cronológicas.
\end{abstract}

Palabras Clave: prospección; relevamiento; espacio; Valle de Ambato.

\begin{abstract}
In this note is in the Valley of Ambato, Catamarca, Argentina. This site was studied with the purpose of deepening the works carried out in the eastern slope of the valley (La Graciana mountain range). In order to achieve this objective, fieldwork (survey and survey) is carried out during the months of October and November 2014. As a result of these activities, registrars are registered and they identify different types of structures (enclosures, corrals and canals), which recover ceramic and lithic materials on the surface. This work allowed to identify an agricultural sector mainly to the agricultural methods that a priori would be in agreement with the proposed productive configuration for the valley. The information obtained from this work suggests that LRA was exclusively focused on agricultural production and that it constituted a particularity within the agro-pastoral model proposed for the area under study. In this way, it is possible to think of this system not as a non-homogeneous whole, but with variants that can respond to social, political, environmental and / or chronological issues.
\end{abstract}

Keywords: prospecting; survey; space; Valley of Ambato.

Las investigaciones arqueológicas desarrolladas en el valle de Ambato han permitido avanzar sobre cómo se fueron configurando las relaciones económicas, sociales, políticas, simbólicas, arquitectónicas y espaciales que permitieron caracterizar a Aguada, durante los siglos VI al XI d.C., como una sociedad internamente diferenciada con diversificación de los roles sociales (Laguens 2004, 2006; Assandri 2007; Fabra 2008; Marconetto 2008; Dantas 2010; Figueroa 2010; Laguens et al. 2013; Figueroa et al. 2016). A partir de esta información se ha podido plantear que para este primer milenio de la era se produce un aumento marcado de la población, divergencias en torno al uso de los espacios públicos y privados y una intensificación de la economía que a su vez incide en cómo se organizaron los recursos (i.e. tierra, agua, animales) (Laguens 2004, 2006). Dentro de los tópicos vinculados a la economía, se propone un sistema inédito para la región basado en una única forma de producción que incluía y articulaba simultáneamente, en un mismo espacio y con una misma infraestructura, la reproducción y cuidado de plantas y animales, constituyendo una práctica integral (Figueroa 2010, Laguens et al. 2013). Dentro de este contexto, lo que se procura en este trabajo es indagar acerca de la viabilidad 
del sistema agro-pastoril propuesto para este lapso temporal, tomando como unidad de análisis el sitio LRA, situado en el extremo sur del de la sierra de La Graciana.

\section{Ubicación del valle y contexto de hallazgo de LRA}

El Valle de Ambato se sitúa en el centro-este de la provincia de Catamarca, dentro del Departamento que lleva el mismo nombre. Este valle se encuentra delimitado hacia el oeste por el cordón montañoso denominado Ambato-Manchao (4.050 msnm), mientras que hacia el este el límite territorial está dado por la sierra GracianaBalcozna (1.850 msnm). En tanto, Los Altos de Singuil y el Valle de Catamarca componen sus fronteras norte y sur respectivamente (Figura 1a).

En lo referente a la biodiversidad, la fauna corresponde a la región zoogeográfica Neotropical, subregión Andino patagónica, con muchos de los representantes de la Provincia Subandina (Cabrera 1976). Mientras que la vegetación se presenta diferenciada en pisos altitudinales, que de acuerdo a Morláns (2009) pueden ser definidos como: Bosque Serrano (entre los 800 y los 1.500 msnm), Arbustal-pastizal (entre los 1.500 hasta los $1.800 \mathrm{msnm}$ ) y Pastizal de altura (por encima de los 1.800- 2.000 msnm).

Un incendio en el año 2009 dejó al descubierto una gran cantidad de materiales y estructuras arqueológicas, lo que permitió identificar un nuevo sitio: La Rinconada Arriba (Figura 1b). Este sitio, constituye, debido a la variedad y cantidad de estructuras observadas, al buen estado de conservación, visibilidad y a lo complejo de su patrón de asentamiento, un excelente escenario para profundizar nuestro conocimiento sobre cómo se configuró el espacio productivo en el valle y discutir acerca de la vigencia del sistema agro-pastoril propuesto oportunamente (Figueroa 2010; Laguens et al. 2013).

\section{Metodología}

El trabajo de campo, consistió en tareas de prospección, recuperación de materiales superficiales y tareas de relevamiento. En cuanto a la técnica de prospección, en LRA optamos por realizar una prospección dirigida, denominada selectiva de ventanas (García Sanjuan 2005). En este tipo de actividad los recorridos se focalizan sobre caminos, senderos, cauces de arroyos, etc., donde las condiciones actuales han eliminado parte de la cobertura vegetal. Asimismo, debido a las características topográficas y vegetacionales del terreno, en dos ocasiones recurrimos a una prospección intensiva mediante muestreo del tipo aleatorio simple (Cerrato Casado 2011). Por último, se realizaron observaciones de imágenes satelitales obtenidas de Google Earth, lo que permitió incluir tres subsistemas agrícolas no identificadas en el terreno.

Respecto a las tareas de relevamiento, se emplearon dos modalidades. En la primera, se utilizó cinta métrica, brújula y GPS con el propósito de indagar sobre las principales características arquitectónicas de las estructuras identificadas (orientación, técnica constructiva, materia prima, altura, espesor, largo y profundidad de los muros). En la segunda, con el fin de poder ubicar espacialmente las construcciones registradas y respetando las características topográficas del terreno, se efectúo un relevamiento planialtimétrico del sitio a partir del empleo de una estación total.

\section{Resultados}

Los resultados obtenidos de estas actividades posibilitaron delimitar al menos 9 sectores en LRA, los cuales fueron denominados LRA1, LRA2, LRA3, LRA4, LRA5, LRA6, LRA7, LRA8 y LRA9 (Figura 2). Precisamente, en LRA1 ubicado a $28^{\circ} 00^{\prime} 54,0^{\prime \prime}$ S y $65^{\circ} 47^{\prime} 26,8^{\prime \prime}$ O y una altura de $1.202 \mathrm{msnm}$, es donde concentramos la mayoría de nuestras tareas. En este sector, de $7.998 \mathrm{~m}^{2}$, registramos 37 estructuras agrícolas del tipo de ladera de contorno ${ }^{1}$,

\footnotetext{
1 Las terrazas de ladera de contorno se caracterizan por poseer muros de contención de piedra que continúan las oscilaciones del terreno (Figueroa 2010).
}

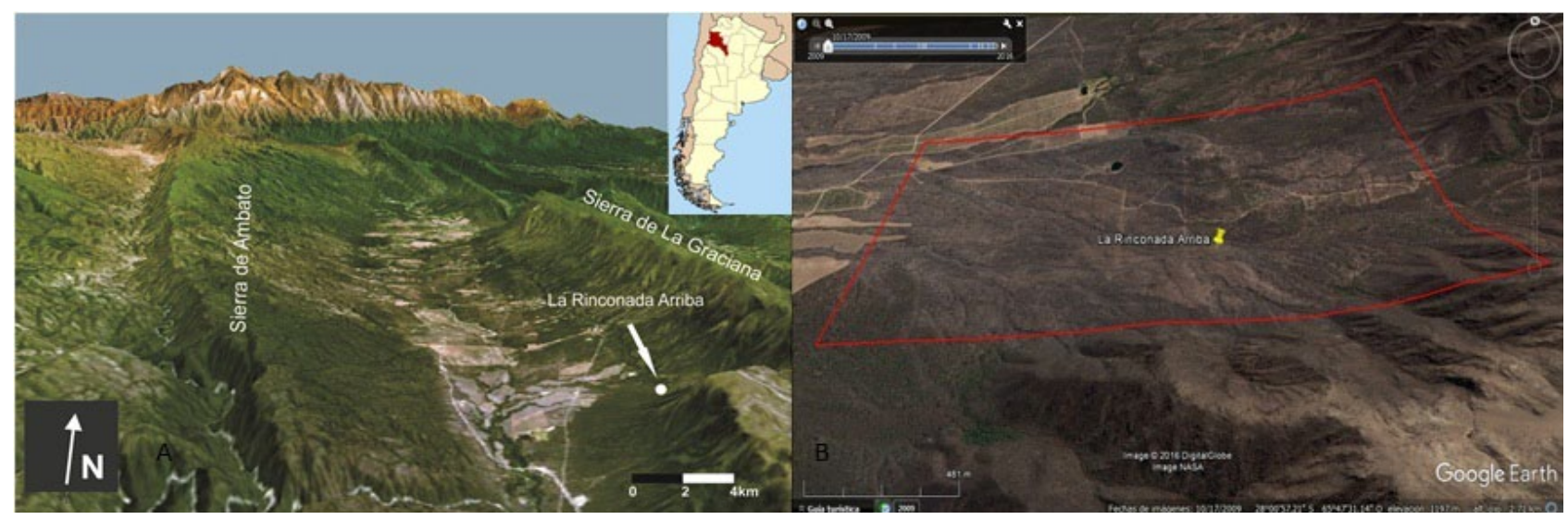

Figura 1: a) Imagen del Valle de Ambato. b) Imagen satelital de La Rinconada Arriba.

Figure 1: a) Image of the Ambato Valley. B) Satellite image of La Rinconada Arriba. 
Figura 2: Imagen satelital de la ubicación de los sectores denominados LRA1, LRA2, LRA3, LRA4, LRA5, LRA6, LRA7, LRA8 y LRA9

Figure 2: Satellite image of the location of the sectors denominated LRA1, LRA2, LRA3, LRA4, LRA5, LRA6, LRA7, LRA8 and LRA9

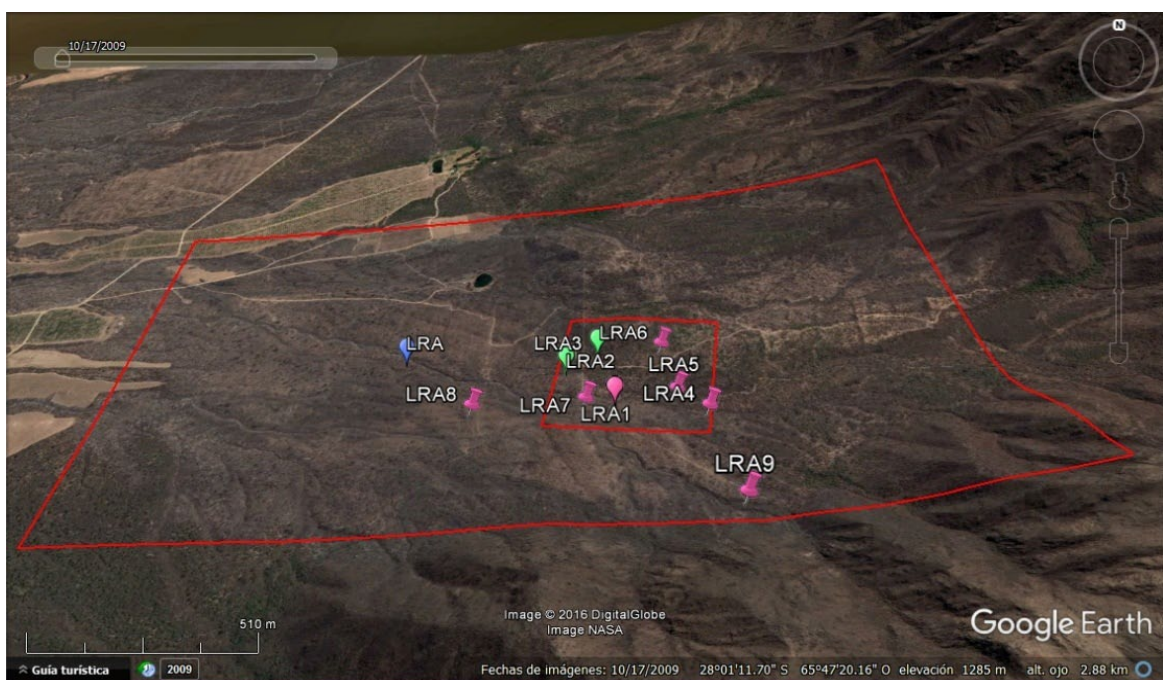

dos de ladera recta², 13 estructuras circulares de muro de piedra simple, posiblemente silos, y dos construcciones rectangulares de muro de piedra simple, las cuales fueron interpretadas, por su similitud con lo morfológico, las técnicas constructivas y los materiales involucrados como canchones (Cruz 2004, Figueroa 2010). No se recuperaron materiales cerámicos, líticos u óseos entre estas estructuras. En LRA1, efectuamos también tareas de relevamiento, así como la excavación de dos terrazas de ladera de contorno.

Una vez prospectado y relevado LRA1 decidimos recorrer zonas aledañas, con el propósito de conocer y reconocer con mayor precisión el alcance las estructuras observadas. Esta actividad se dividió en dos sectores de trabajo, el primero denominado LRA2 y el segundo LRA3. En ambos casos, implementamos una prospección del tipo intensiva mediante muestreo del tipo aleatorio simple (Cerrato Casado 2011).

LRA2 se encuentra ubicado hacia el norte, a $195 \mathrm{~m}$ de distancia de LRA1 y las coordenadas del mismo son $28^{\circ} 0^{\prime}$ 49,02" Sy $65^{\circ} 47^{\prime} 31,77^{\prime \prime} \mathrm{O}$. El área de prospección cubrió un total de $11.620 \mathrm{~m}^{2}$ y logramos recuperar por medio de recolecciones superficiales 41 fragmentos cerámicos y 11 instrumentos líticos. En tanto que, no registraron estructuras agrícolas, habitacionales, pastoriles o hídricas.

Por su parte, LRA3 se halla situado a $230 \mathrm{~m}$ al noroeste de LRA1 y sus coordenadas geográficas son $28^{\circ} 0^{\prime} 46,73^{\prime \prime} \mathrm{S}$ y $65^{\circ} 47^{\prime} 28,52^{\prime \prime} \mathrm{O}$. En este caso, en un área de $12.054 \mathrm{~m}^{2}$, identificamos cuatro recintos, uno rectangular de muro de piedra simple (LRA3R1), otro semicircular de muro de piedra doble (LRA3R2) y dos indeterminados (LRA3R3 y LRA3R4). LRA3R1, con orientación este-oeste, posee $13 \mathrm{~m}$ de largo y 6,30 $\mathrm{m}$ de ancho. Su posicionamiento geográfico es $28^{\circ} 00^{\prime} 45,10^{\prime \prime} \mathrm{S}$ y $65^{\circ} 47^{\prime} 29,52^{\prime \prime} \mathrm{O}$ y de su interior recuperamos en superficie ocho fragmentos

2 Las terrazas de ladera recta corresponden a terrazas que poseen muros de contención de piedras rectilíneos, los cuales cortan de forma transversal la pendiente (Figueroa 2010). cerámicos. LRA3R2 es de forma semicircular, de 6,30 $\mathrm{m}$ por $6 \mathrm{~m}$. Su posicionamiento geográfico es $28^{\circ} 00^{\prime}$ $45^{\prime \prime} \mathrm{S}$ y $65^{\circ} 47^{\prime} 29,03^{\prime \prime}$ O y en su interior recobramos tres fragmentos cerámicos. LRA3R3, se encuentra aproximadamente a $8 \mathrm{~m}$ de LRA3R2 Y: Su ubicación es de $28^{\circ} 0^{\prime} 44,46^{\prime \prime}$ 'S y $65^{\circ} 47^{\prime} 29,39^{\prime \prime}$ O. No fue factible identificar su forma debido a la destrucción que presentaba el recinto. Por su parte, LRA3R4 se encuentra a $67 \mathrm{~m}$ de LRA3R1 y su ubicación geográfica es $28^{\circ} 0^{\prime}$ $43,07^{\prime \prime}$ S y $65^{\circ} 47^{\prime} 30,00^{\prime \prime}$ O, el mismo se encontraba con un grado de erosión elevado y una destrucción importante producto del accionar de los animales locales.

Prácticamente adosado a LRA3R1 registramos un tramo de canal (LRA3C1) construido mediante la utilización de piedras clavadas de canto en la tierra. Las tareas de relevamiento efectuadas sobre este último permitieron determinar que esta estructura cuenta con 8,30 m de largo y $35 \mathrm{~cm}$ de ancho. Además, pudimos establecer que no se asocia a ninguna terraza de cultivo, sino que se dirige hacia el fondo del valle donde se emplazan los núcleos aldeanos. LRA3C1 posee una orientación este-oeste y sus coordenadas geográficas son $28^{\circ} 0^{\prime \prime}$ $45,87^{\prime \prime}$ S y $65^{\circ} 47^{\prime} 28,43^{\prime \prime} \mathrm{O}$. Su rumbo es de $260^{\circ}$. En estrecha proximidad de estas estructuras se recuperaron en superficie, en forma aislada o como conjuntos de hallazgos, 127 fragmentos cerámicos y 21 instrumentos líticos.

Asimismo, producto de las prospecciones realizadas, a $170 \mathrm{~m}$ hacia el este de LRA1 pudimos registrar tres subsistemas o concentraciones de terrazas de cultivo, denominadas, LRA4, LRA5 y LRA6, todas ellas emplazadas en medio de una densa vegetación que impidió determinar fehacientemente su cantidad. La concentración de terrazas de cultivo LRA4 se ubica a $28^{\circ} 00^{\prime} 56,09^{\prime \prime}$ S y $65^{\circ} 47^{\prime} 18,08^{\prime \prime} \mathrm{O}$, a 1.202 msnm. La misma presenta una orientación este-oeste y consiste en 10 terrazas de ladera de contorno. En sus inmediaciones, también se observaron varias estructuras circulares de muro de piedra simple que en algunos casos comparten 
sus paredes con las terrazas. LRA5 se encuentra a $28^{\circ}$ $00^{\prime} 53,5^{\prime \prime} \mathrm{S}$ y $65^{\circ} 47^{\prime} 21,5^{\prime} \mathrm{O}$, a una altura de 1.197 msnm y a $118 \mathrm{~m}$ al noreste de LRA1. Comparte el mismo sentido de orientación que LRA4. En este caso, la ubicación de LRA5 es sobre una escorrentía estacional y se tratarían de al menos dos terrazas de ladera rectas. La concentración restante, LRA6, se localiza a $240 \mathrm{~m}$ al noreste de LRA1, exhibe una orientación sur-sureste y se sitúa a 1.224 msnm, siendo sus coordenadas geográficas $28^{\circ} 0^{\prime} 47.07^{\prime \prime} \mathrm{S}$ y $65^{\circ} 47^{\prime} 22.30^{\prime \prime} \mathrm{O}$. Aquí, de modo similar a lo ocurrido en los casos anteriores y debido a la espesa vegetación que las rodea, sólo se lograron registrar tres terrazas de contorno que presentaban además un alto grado de erosión.

Finalmente, a partir de observaciones satelitales pudimos identificar tres subsistemas agrícolas más, los cuales fueron denominados: LRA7, LRA8 y LRA9. El subsistema LRA7, $28^{\circ} 0^{\prime} 54,30^{\prime \prime} \mathrm{S}$ y $65^{\circ} 47^{\prime} 30,08^{\prime \prime}$ O, se ubica a $158 \mathrm{~m}$ de distancia de LRA1, aquí se reconocieron 10 estructuras agrícolas con orientación este-oeste. En promedio, su longitud es de $15 \mathrm{~m}$ y exhiben una distancia entre terrazas que va desde los 5 a los $10 \mathrm{~m}$. El subsistema LRA8, por su parte, se encuentra ubicado a $28^{\circ} 0^{\prime} 54,69^{\prime \prime} \mathrm{S}$ y $65^{\circ} 47^{\prime} 41,33^{\prime \prime} \mathrm{O}$, a una distancia de 387 m de LRA1, hacia el oeste. Este subsistema cuenta con 8 terrazas que tienen una orientación este-oeste. La longitud promedio de estas estructuras es de 36,5 m y poseen una distancia menor a $7 \mathrm{~m}$, aproximadamente. Por último, el subsistema LRA9, se encuentra a 488 m de LRA1 hacia el sudeste, cuenta con 4 terrazas de orientación noreste-suroeste. Sus coordenadas geográficas son $28^{\circ}$ 1' $8,52^{\prime \prime}$ S y $65^{\circ} 47^{\prime} 16,20^{\prime \prime} \mathrm{O}$. El promedio de distancia entre terrazas es de 6,85 m, y el de longitud de $11 \mathrm{~m}$. En todos los casos (LRA7, LRA8 y LRA9) las estructuras agrícolas corresponden a las denominadas terrazas de ladera de contorno.

A partir de los trabajos de prospección se pudo observar que las estructuras agrícolas relevadas poseen la misma técnica constructiva, que consisten en piedras apiladas combinadas sin preferencia de tamaños, formas ni clases. En este sentido, se pudo constatar que las piedras utilizadas, tales como esquisto, migmatita y gneis ${ }^{3}$ pueden ser rectangulares, cuadrangulares, triangulares y hasta aproximadamente esféricas. Asimismo, no se logró observar que hayan sido trabajadas pero sí elegidas intencionalmente para encajar y trabar, ya que no se registró el uso de ningún tipo de mortero o amalgama. Las terrazas registradas poseen una orientación esteoeste y una longitud que varía entre 5 y $60 \mathrm{~m}$. Su ancho oscila entre 30 y $60 \mathrm{~cm}$ y su altura fluctúa entre 35 y $50 \mathrm{~cm}$, en tanto que la distancia entre muros es de aproximadamente $5 \mathrm{~m}$. En definitiva, a partir de la información recabada podemos afirmar que 65 de las 67 terrazas registradas pertenecen a las denominadas

\footnotetext{
3 Es posible encontrar este tipo de material y técnica constructiva en el resto del valle (Figueroa 2010).
}

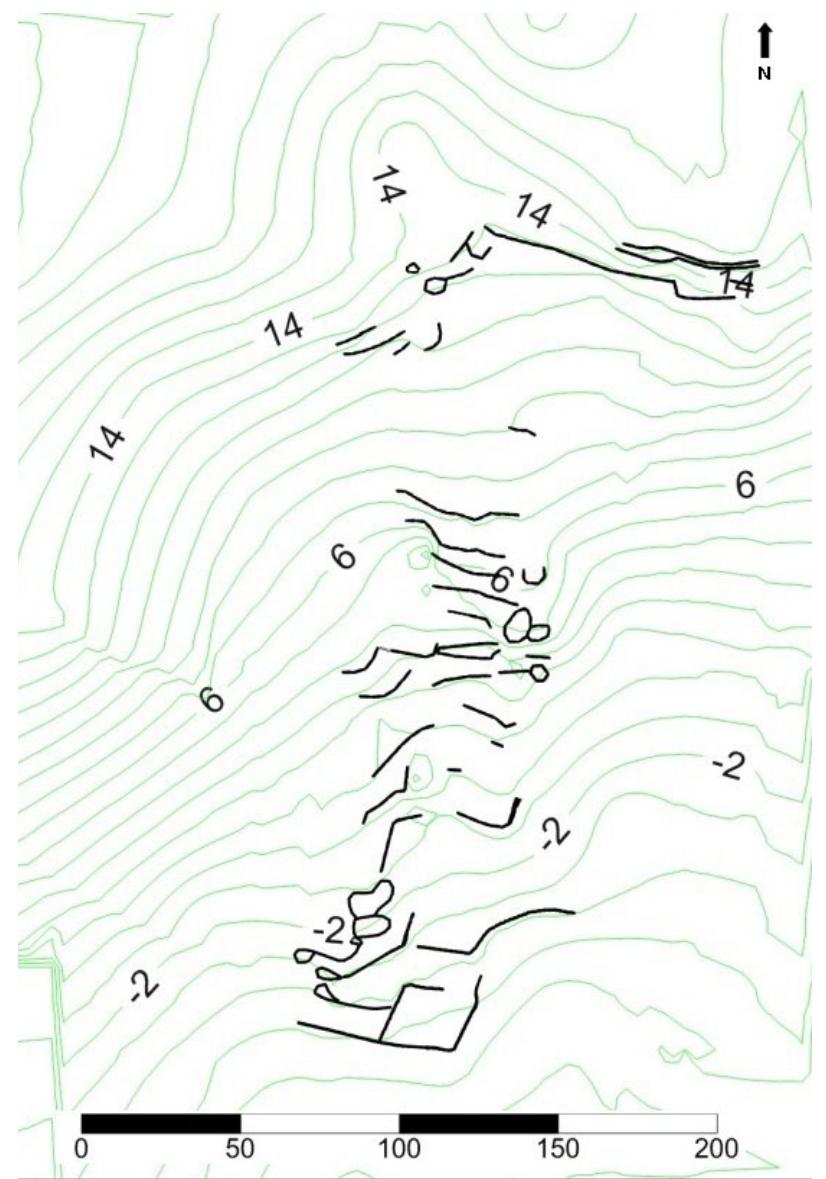

Figura 3: plano altimétrico del sitio LRA1

Figure 3: Altimetric plane of the LRA1 site

terrazas de contorno y dos a las rectas.

Por otro lado, en base a los datos obtenidos en el relevamiento efectuado con la estación total y la utilización del programa informático Surfer 10 , confeccionamos un plano de LRA1, que representa aproximadamente un 20 $\%$ de la totalidad de LRA. A través del análisis del mismo, pudimos dar cuenta de la posición espacial de las terrazas de cultivo, las características de su trazado y cómo estas se vinculan con otras clases de estructuras, así como con el terreno en el cual fueron emplazadas (Figura 3).

En la Figura 3, puede observarse como las terrazas de ladera de contorno siguen las oscilaciones del terreno. El plano, a su vez, nos permitió visualizar diversas asociaciones entre las estructuras registradas. De este modo, podemos mencionar la presencia de 13 recintos circulares con muro de piedra simple distribuidos entre las terrazas de contorno. Tres de estas construcciones se encuentran directamente adosadas a ellas, conformando agrupaciones de hasta tres estructuras. Las construcciones circulares tienen un diámetro promedio de 5,65 m y no se recobró material cerámico o lítico asociado a ellas (Figura 4a, 4b y 4c).

Además, registramos dos rectángulos de muro de 


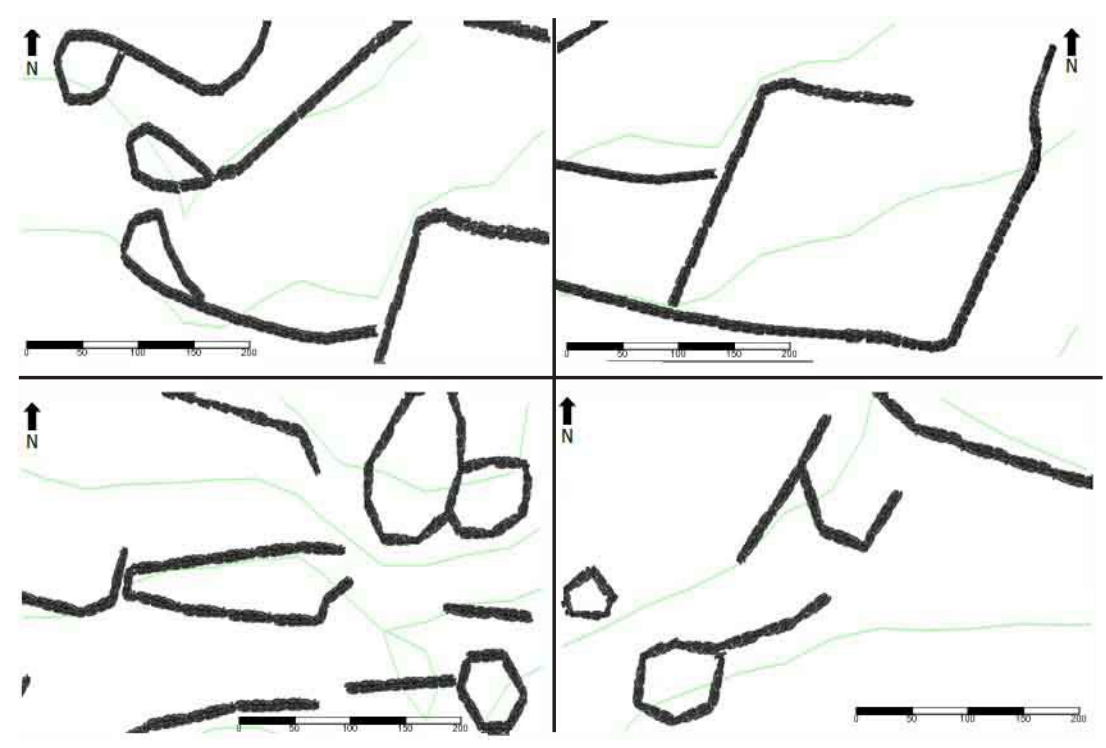

Figura 4: Diversas asociaciones de estructuras. A) recintos circulares de muro de piedra simple compartiendo muros. B) Recintos circulares de muros de piedra simple compartiendo muro con las terrazas de contorno. C) Estructuras circulares/ ovaladas ubicadas en extremos de las terrazas. D) Estructura rectangular de piedra de muro simple

Figure 4: Various associations of structures. A) Circular enclosures of simple stone wall sharing walls. B) Circular enclosures of simple stone walls sharing a wall with the contour terraces. C) Circular / oval structures located at the ends of the terraces. D) Rectangular structure of stone of simple Wall

Tabla 1: Material cerámico recuperado en LRA2 y LRA3

Table 1: Ceramic material recovered in LRA2 and LRA3

\begin{tabular}{lcccc}
\hline & & \multicolumn{3}{c}{ Parte de la Vasija } \\
Clase Tecnológica & Número de Fragmentos & Cu & Ba & Bo \\
\hline Negro Pulido & $4(3,15 \%)$ & 1 & 3 \\
Rojo Pulido & $8(6,23 \%)$ & 6 & 1 & 1 \\
& $2(2,36 \%)$ & & & 2 \\
Naranja Alisado & $17(13,39 \%)$ & 16 & 1 \\
Rojo Alisado/pulido & $4(3,15 \%)$ & 4 & & \\
castaño Alisado/Pulido & $2(1,57 \%)$ & 2 & & \\
Castaño Pulido & $93(73,23 \%)$ & 92 & 1 \\
Castaño Alisado & & & & \\
\hline Tosca & $38(29,92 \%)$ & 35 & 1 & 2 \\
\hline & $168(100 \%)$ & 156 & 2 & 10 \\
\hline
\end{tabular}

piedra simple que posiblemente se tratarían de cuadros o canchones de cultivo (Figura 4d). Generalmente, la bibliografía del NOA, señala que estas construcciones se localizan frecuentemente en los sectores próximos al fondo del valle y que presentan en su mayoría irrigación artificial (Albeck 1993; Cruz 2004, 2006; entre otros). Sin embargo, en nuestro caso, la diferencia radica, por un lado, en que no se identificaron estructuras hídricas destinada al riego de las mismas y, por otro, que las estructuras comparten muro con las terrazas de cultivo o bien se sitúan en estrecha cercanía a ellas.
En relación al material recuperado producto de los trabajos de prospección, la alfarería, toda ella fragmentaria, ascendió a un número de 168 fragmentos, logramos establecer ocho grandes modos o clases tecnológicas (Sensu Fabra 2008), todas vinculadas a ocupaciones Aguada en el valle, las que repiten su presencia en LRA2 y LRA3, a excepción de la denominada Naranja Alisado que sólo fue registrado en LRA3. Más allá de esta diferencia, en general pudimos determinar que el modo tecnológico Castaño Alisado es el que se encuentra con mayor frecuencia en la toda zona trabajada (57\%), mientras que la clase denominada Tosca es la segunda con mayor representación (23\%). Los modos tecnológicos menos usuales son el Naranja Alisado (1\%), Negro Pulido (2\%) y Rojo Pulido (4\%) (Tabla 1). Mientras que, el material lítico consiste en 32 instrumentos y desechos de talla, los que actualmente se hallan en proceso de estudio.

El cuerpo de datos obtenidos hasta el momento (clases de estructuras, tipo de emplazamiento, técnica constructiva, material cerámico y lítico recobrado, etc.) nos permite situar, mediante el empleo de una cronología relativa (Giovannetti et al. 2010), a LRA, en algún momento de la ocupación Aguada en el valle.

\section{Discusiones y consideraciones finales}

Teniendo en cuenta el modelo productivo propuesto para el Valle de Ambato (Figueroa 2010, Laguens et al. 2013, entre otros), retomaremos la idea que guió esta investigación. La misma estuvo centrada en analizar cómo se configuró este espacio que denominamos LRA ubicado en el sector sur de la sierra de La Graciana, durante los siglos VI al XI d.C. La labor llevada a cabo en el lugar, nos permitió registrar similitudes y diferencias en el espacio ${ }^{4}$,

\footnotetext{
4 Se tomarán en cuenta los aportes en torno a las tres reflexiones epistemológicas sobre el concepto de espacio expuestos oportunamente por Criado Boado (1995). La primera, postula que el espacio no es una entidad física dada, estática, inerte, sino más bien está en continuo movimiento, es creada y re-creada. La segunda, se encuentra
} 
que estarían marcando ciertas particularidades con el modelo mencionado anteriormente.

En este sentido, un rasgo propio de LRA, a diferencia de lo observado en ambas vertientes del valle, es que los recintos identificados son escasos -una vivienda (LRA3R1), un corral (LRA3R2) y dos indeterminados (LRA3R3 y LRA3R4)- y no se asocian de modo contiguo a las estructuras productivas. Un ejemplo de ello es lo registrado por Figueroa (2010) en el sitio Los Varela 2, donde es posible observar como las estructuras agrícolas y pastoriles se encuentran compartiendo muro, tal como puede visualizarse en los círculos rojos de la Figura 5. En tanto, las estructuras habitacionales, ubicadas dentro de los círculos amarillos, por lo general no distan a más de un centenar de metros de los aterrazamientos (Figura

vinculada a la relación entre espacio, pensamiento y sociedad. Aquí, la construcción del espacio social aparece como una parte esencial del proceso social, que está determinado por un sistema de saber que es compartido con la organización socio-económica y con la definición de individuo vigente en ese contexto. Finalmente, la tercera menciona que el concepto de espacio está cargado de significaciones que cobran sentido en un contexto determinado, no obstante, su abordaje, permite esbozar reflexiones o hipótesis sobre como el mismo pudo haber sido experimentado en culturas diferentes a las occidentales.

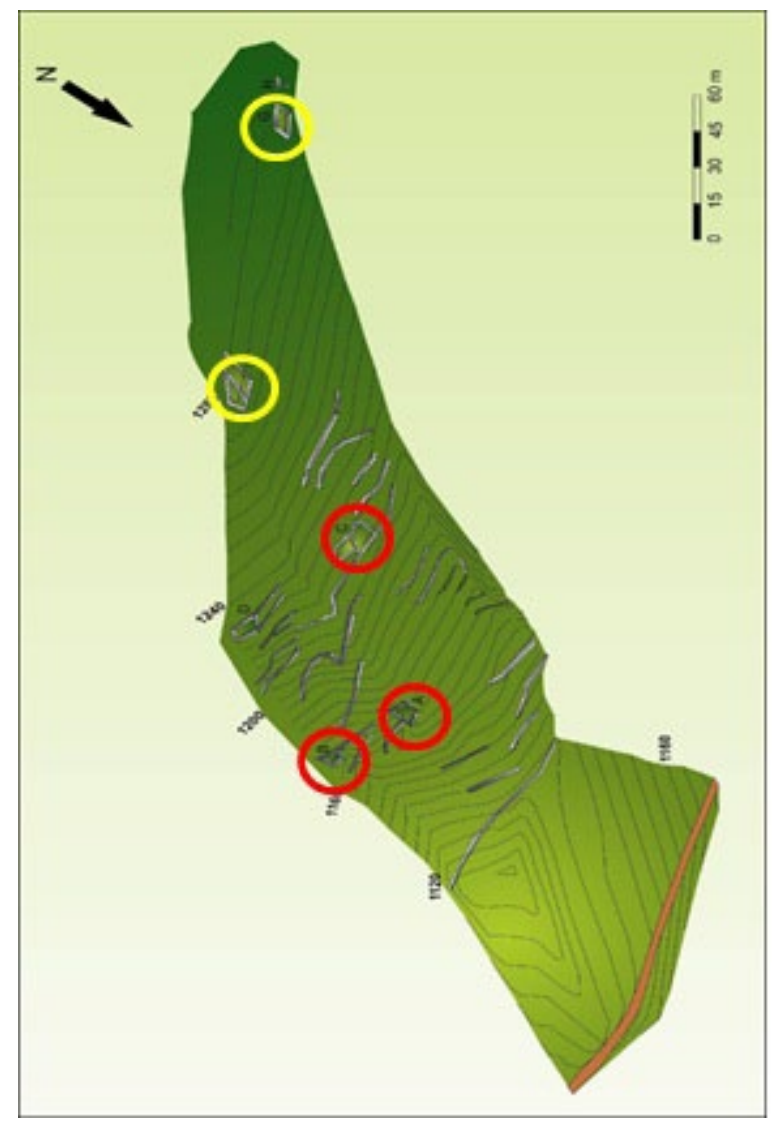

Figura 5: relevamiento planialtimétrico de recintos y terrazas de contorno. Concentración Los Varela 2. Extraído de Figueroa 2010

Figure 5: planialtimetric survey of enclosures and contour terraces. Concentration Los Varela 2. Extracted from Figueroa 2010
5) (Dantas y Figueroa 2009, Figueroa 2013, Laguens et al. 2013). Estos datos nos hacen pensar nuevamente en la disposición de las estructuras de LRA, donde la vivienda más próxima de LRA1 es la ubicada en LRA3 a $230 \mathrm{~m}$. Esta información nos permite dar cuenta que el espacio productivo y los sitios habitacionales, si bien se encuentran en cercanías a las terrazas de contorno habitando el mismo espacio productivo, no se hallan compartiendo sus muros en forma de medianera.

A su vez, fue posible registrar en LRA1 dos estructuras rectangulares de muro de piedra simple (Figura 3), que posiblemente fueron utilizados como canchones de cultivo, los cuales hasta el momento, sólo habían sido registrados por Cruz (2004). Sin embargo, lo llamativo en LRA1 es que estas construcciones comparten muro con las terrazas de cultivo y con numerosos $(\mathrm{N}=13)$ recintos circulares de muro de piedra simple (Figura 3 y 4 d). En tanto, también pudimos constatar que el ancho de las terrazas de contorno de LRA1 posee un promedio de 5 $\mathrm{m}$, a diferencia de las situadas en la vertiente occidental donde este promedio desciende a 3,31 m.

Por otro lado, entre las similitudes observadas con el resto del valle, tal como se señaló en párrafos anteriores, los recintos, las terrazas de cultivo, el canal y la estructura ganadera de LRA fueron edificados con igual técnica constructiva y materia prima que lo documentado para el resto del valle (Figueroa 2010, Figueroa et al. 2016). Además, los subsistemas de producción de LRA al igual que los ubicados en ambas vertientes, habrían estado articulados estrechamente con las unidades residenciales emplazadas en el fondo del valle, tal como ya fuera postulado por Figueroa en trabajos previos (2010, 2013, etc.). Mientras que el material cerámico recuperado y analizado en LRA, al igual que el registrado para el resto de ambas laderas, corresponde en su totalidad a Aguada.

En definitiva, toda esta información nos presenta a priori un registro que pareciera contrarrestar con el modelo intensivo e integrado propuesto para el valle y nos lleva a pensar que LRA fue destinado sólo a la actividad agrícola. Sin embargo, debemos considerar que el sistema agro-pastoril integrado no fue un todo homogéneo, por el contrario el registro nos estaría marcando que bajo este sistema conviven o existen diversas formas de generar la producción de alimentos, y que esas formas se deben quizás a prácticas sociales, políticas, ambientales, cronológicas, que pueden ir cambiando en función de responder particularidades y/o urgencias. Precisamente, este panorama nos permite reflexionar y discutir sobre las variaciones del modelo agro-pastoril propuesto para el valle de Ambato y a la vez nos abre un gran interrogante: ¿A qué se deben estas particularidades identificadas en LRA?

Creemos, que para poder avanzar en las respuestas a este interrogante, el cual excede los límite de 
este trabajo, es necesario continuar con esta línea investigativa, ampliando tanto los trabajos de campo (prospecciones, relevamientos y excavaciones) como de laboratorio (nuevos estudios de microfósiles en terrazas y canchones, análisis físico-químico del suelo, estudios de los artefactos recobrados, análisis de isotopos, fechados radiocarbónicos, etc.). Además, resulta indispensable comenzar a integrar los resultados alcanzados en Ambato con los obtenidos por parte de otros investigadores en regiones próximas. Sólo de este modo, aportando nuevos datos a las investigaciones ya realizadas, podremos reflexionar más detenidamente en relación a las prácticas productivas implementadas por las sociedades aguadas en la zona bajo estudio.

Córdoba, 21 de Marzo de 2017

\section{Agradecimientos}

Este estudio fue financiado por subsidios de la Secretaría de Ciencia y Tecnología de la Universidad Nacional de Córdoba y por las Becas de Estímulo a la Vocación Científica del Consejo Interuniversitario Nacional (EVC CIN) durante el 2015-2016 y su extensión hasta el 2017.

\section{Bibliografía}

Albeck, M. E. 1993. Contribución al estudio de los sistemas agrícolas prehispánicos de casabindo (puna de Jujuy). Tesis de doctorado, área antropología, Facultad de Ciencias Naturales y Museo, Universidad Nacional de La Plata, La Plata. ms.

Assandri, S. B. 2007. Procesos de complejización social y organización espacial en el Valle de Ambato, Catamarca, Argentina. Tesis de maestría en arqueología. Universidad Internacional de Andalucía, España.

Cabrera, A. 1976. Regiones fitogeográficas argentinas. Enciclopedia argentina de agricultura y jardinería, vol. 1. ACME, Buenos Aires.

Cerrato Casado, E. 2011. La prospección arqueológica superficial. Un método no destructivo para una ciencia que sí lo es. Arte, arqueología e historia 18, 151-160.

Criado Boado, F. 1995. límites y posibilidades de la arqueología del paisaje. Revista de prehistoria y arqueología (2) : 9-55.

Cruz, P. J. 2004. Archéologie de la mort dans la Vallée d'ambato. Homme et milieu dans le bassin de Los Puestos (Catamarca-Argentine) durant la période d'intégration régionale (IVE-XE siècles après j.-C.). Tesis de doctorado, Universidad de Paris i Pantheonsorbonne, Paris. ms.

Cruz, P. J. 2006. Complejidad y heterogeneidad en los andes meridionales durante el período de integración regional (siglos IV-X d.C.). Nuevos datos acerca de la arqueología de la cuenca del río de Los Puestos (dpto. Ambato - Catamarca, Argentina). Bulletin de l'ifea 35 (2): 121-148.

Dantas, M. 2010. Arqueología de los animales y procesos de diferenciación social en el Valle de Ambato, Catamarca, Argentina. Tesis de doctorado, Facultad de Filosofía y Humanidades, Universidad Nacional de Córdoba, Córdoba. ms.

Dantas, M., G. G. Figueroa. 2009. Terrazas y corrales como espacios integrados de producción agro-pastoril en el Valle de Ambato, Catamarca, Argentina (S. VI-XI d.C.). relaciones de la sociedad argentina de antropología XXXIV: 343-350.

Fabra, M. 2008. Producción tecnológica y cambio social en sociedades agrícolas prehispánicas (Valle de Ambato, (atamarca). Bar International Series. Oxford.

Figueroa, G. G. 2010. "organización de la producción agrícola en contextos sociales no igualitarios: el caso del Valle de Ambato, Catamarca, entre los Siglos VII y XI d. C". Tesis de doctorado, Facultad de Filosofía y Humanidades, Universidad Nacional de Córdoba, Córdoba. ms.

Figueroa, G. G. 2013. Estrategias productivas en aguada de Ambato (Catamarca, Argentina). Relaciones de la Sociedad Argentina de Antropología (XXXVIII): 111-135.

Figueroa, G. G., M. Rodríguez Oviedo, M. dantas, A. Laguens. 2016. Investigaciones arqueológicas en el sitio la rinconada arriba, Valle de Ambato, Catamarca (siglos VI al XI d.C). Revista de Antropología del Museo de Entre Ríos 2 (1), 1-12.

García Sanjúan, L. 2005. Introducción al reconocimiento y análisis arqueológico del territorio. Editorial Ariel, Barcelona.

Giovannetti, M., M.C. Páez, G. Cochero, P. Espósito, G. Corrado, J. Spina, y F. Franchetti. 2010. Sectorización del espacio, cerámica y cronología relativa. Análisis comparativos en un asentamiento agrícola multicomponente (LoS Colorados, Catamarca) en La arqueometría en argentina y latinoamérica, editado por Silvana Bertolino, Roxana Cattáneo y Andrés Izeta, pp. 55-60.

Laguens, A. 2004. Arqueología de la diferenciación social en el Valle de Ambato, Catamarca, Argentina (S. II-VI d.C.): el actualismo como metodología de análisis. Relaciones de la Sociedad Argentina de Antropología XXIX: 137-161.

Laguens, A. 2006. Continuidad y ruptura en procesos de diferenciación social en comunidades aldeanas del Valle de Ambato, Catamarca, Argentina (S. IV-X d.C.). Chun- 
gara, Revista de Arqueología Chilena 38 (2): 211-222.

Laguens, A., G. G. Figueroa y M. Dantas. 2013. Tramas y prácticas agro-pastoriles en el Valle de Ambato, Catamarca (siglos VI y XI d.C.). Revista Arqueología 19 (1): 131-152.

Marconetto, M. B. 2008. Recursos forestales y el proceso de diferenciación social en tiempos prehispánicos en el Valle de Ambato, Catamarca, Argentina. British Archaeological Reports s1785, South American Archaeology Series $n^{\circ} 3$, Oxford.

Morláns, M. C. 2009. Regiones naturales de Catamarca. Provincias geológicas y provincias fitogeográficas. Editorial Científica Universitaria - Universidad Nacional de Catamarca. 